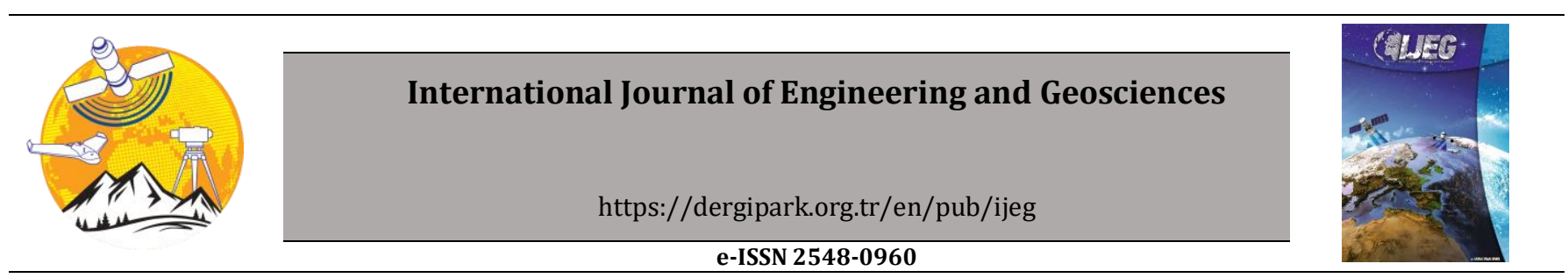

\title{
Clutter removal in millimeter wave GB-SAR images using OTSU's thresholding method
}

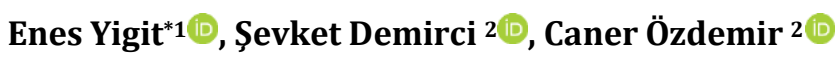 \\ ${ }^{1}$ Bursa Uludağ University, Engineering Faculty, Department of Electrical Electronics Engineering, Bursa, Turkey \\ ${ }^{2}$ Mersin University, Engineering Faculty, Department of Electrical Electronics Engineering, Mersin, Turkey
}

\author{
Keywords \\ Synthetic Aperture Radar \\ OTSU \\ Back projection Algorithm \\ Clutter Cancellation
}

\begin{abstract}
In this study, a filtering method based on the threshold value of normalized synthetic aperture radar (SAR) data is proposed to eliminate clutter in millimeter wave ground based synthetic aperture radar (GB-SAR) images. In the proposed method, first, stepped frequency continuous wave SAR data are reconstructed by using the back-projection algorithm and focused complex SAR data are obtained. Then, the amplitude values of the complex SAR data are normalized and the best threshold values to distinguish the target from clutter is determined by the OTSU's thresholding method. Next, a filter mask is created that cancels all data below the computed threshold values. The mask matrix is finally multiplied with the resulted GB-SAR data to eliminate all clutter from the image. With the proposed technique, the best threshold value is determined automatically by directly processing the raw data without converting the SAR data into any RGB images. The proposed technique is validated through real GB-SAR experiments that were carried out in the frequency band of 78-81 GHz. In the experiments, challenging GB-SAR data are obtained using high cluttered background materials, and very successful filtering operations are performed with the proposed technique.
\end{abstract}

\section{INTRODUCTION}

Synthetic aperture radar (SAR) is a highly effective remote sensing tool in producing high resolution images of moving and fixed land targets. While traditional SAR systems have been typically installed on satellites and aircrafts, they also have been applied to near-surface unmanned aerial vehicles and terrestrial vehicles in recent years. Compared to air-based SAR, ground-based (GB) SAR systems offer very cheap and practical implementations and thus find applications in many fields such as through the wall imaging (Engin et al., 2007), agricultural applications (Gomez et al., 2006), observing unstable slopes (Pieraccini et al., 2006), foreign object debris detection (Yigit et al., 2012; Beasley et al., 2004) and concealed object detection (Jaeger et al., 2007; Demirci et al., 2012). Among these, millimeter wave (MMW) SAR (Yigit et al., 2012; Jaeger et al., 2007; Demirci et al., 2012) applications, as providing very high resolution images, have become very popular in recent years. However, removal of clutter echoes in both traditional SAR images and millimeter wave (MMW) GBSAR images are one of the most important problems encountered in target detection. This problem becomes more intense in high frequency and broadband MMW SAR images. Many studies have been carried on to eliminate different types of unwanted clutter from focused SAR images of various Earth regions (Freitas et al., 2005; Conte et al., 1991). Although the popular methods based on statistical modeling and Constant False Alarm Rate (CFAR) [Demirci et al., 2008] are frequently used for clutter removal, clutter has not been completely eliminated due to the tradeoff encountered in setting the CFAR window (Toktas et al.2017). When the large CFAR window is selected, much information about the target remains in the image, while there are still clutter residues in the background. When the window is selected small, the background is mostly cleared, but there is a loss of target information. However, advanced image processing techniques can be used effectively to overcome this trade-off problem. In this study, the OTSU method, which is highly effective in gray level filtering, has been proposed to eliminate unwanted clutter in MMW SAR images. Although OTSU method is an effective method in image processing, it is mostly used in HoughSaturation-Value (HSV) regions of (Red, Green, and Blue) RGB images (Sabancl et al.2018). In many radar imaging applications where the OTSU method was used, the 
operations were executed through the RGB image files (Işıker and Özdemir, 2019; Ișıker et al., 2018; Işıker et al., 2015; Khoukhi et al.2019; $\mathrm{Lu}$ and $\mathrm{Hu}$ 2012). However, converting SAR data into image files and then filtering these files again with image processing causes high computation time and data loss. Thanks to the method proposed in this study, a practical but effective method for filtering raw SAR data is presented. With the proposed method, images with a low signal-to-clutter ratio, such as MMW-SAR data, were precisely cleaned. To assessment the success and performance of the technique, 2 different measurements were performed at MMW frequencies (in the range of 78-81 GHz) and visual comparisons were made by focusing on each GB-SAR data with the back projection algorithm (BPA) (Yigit et al., 2013; Yigit et al., 2020) in order to comprehend only the filter performance.

The main sections of the study are as follows: In the second part, the SAR imaging and focusing method is briefly presented, while the proposed filtering method is given in detail. The experimental organization and the outcomes of the measurements are given in the third section. In the last section, the results of the study are evaluated and the study is summarized.

\section{METHOD: SAR IMAGE RECONSTRUCTION AND OTSU METHOD}

\subsection{GB-SAR IMAGE RECONSTRUCTION}

Fig. 1 shows the setup of a simple GB-SAR scanning system. In a 2 dimensional (2D) imaging geometry, the reflection function of the targets in the scanning region is defined as $\rho(x, y)$ and the positions of the antennas are expressed in Cartesian coordinates as $(x, y)=$ $\left(D_{p} \sin \beta, D_{p} \cos \beta\right)$ where, $D_{p}$ and $\beta$ are the distance and angle between antennas and origin of the scene, respectively. Assuming that there are a total of $\mathrm{P}$ point scatterers in the scanned region, the backscatter signal obtained by the radar can be expressed as follows.

$S_{\beta}(k)=\sum_{p=1}^{P} \rho_{p} \mathrm{e}^{-j k D_{p}(\beta)}$

where, $k=4 \pi f / c$ is a (two way) wave number, $f$ and $c$ are ferquency and speed of the light, respectively.

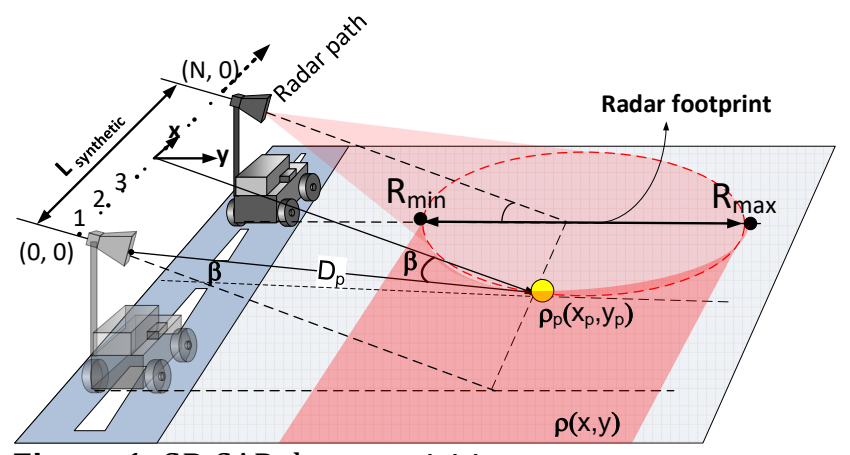

Figure 1. GB-SAR data acquisition geometry

The instantaneous distance between the antennas and targets,

$D_{p}(\beta)=\sqrt{\left(x-x_{p}\right)^{2}+\left(y-y_{p}\right)^{2}}$ where, $\left(x_{p}, y_{p}\right)$ and $(x, y)$ correspond to coordinate of the $\mathrm{p}$ th scatterer and antennas, respectively.

As seen in the Fig. 1, the SAR platform collects back scatter data at a total of $\mathrm{N}$ different points along the $\mathrm{L}$ synthetic aperture and obtains 2D GB-SAR data (Yigit et al., 2013; Yigit et al., 2020). Then, in order to transform the obtained raw SAR data into a meaningful image, focused complex SAR data $\rho(x, y)$ is obtained using any reconstruction algorithm such as BPA (Yigit et al., 2013).

\subsection{OTSU BASED FILTERING ALGORITHM}

OTSU is an effective method especially used in automatic filtering of gray level images (Otsu, N., 1979). This method is mostly preferred for picture data in RGB format. For this reason, focused SAR data must first be converted into RGB image format and then processed into a suitable format such as HSV for OTSU filtering by additional processing (Işıker and Özdemir, 2019). However, this process adds additional cost and the success of the filter depends on the resolution of the SAR image. This is also a disadvantage for the filtering process. In this study, a filtering method based directly on focused raw SAR data rather than using any image format is proposed. The normalized filter method first starts with obtaining the amplitude $|\rho|$ of the complex $\rho(x, y)$ matrix.

Using Eq. 3, the values of the $|\rho|$ data are normalized to be between 0 and 1 .

$\rho_{n}=\frac{|\rho|-\min |\rho|}{\max |\rho|-\min |\rho|}$

In the $\rho_{n}$ data, the values of target region are mostly close to 1 , while the values in the non-target regions are close to 0 . In order to clean the background clutter precisely, the mean value is subtracted from $\rho_{n}$ data.

$\rho_{m}=\rho_{n}-\operatorname{mean}\left(\rho_{n}\right)$

The values of the data obtained by Eq. 4 which are less than zero are equalized to 0 and $\rho_{m}$, data is obtained. This data is then normalized as follows.

$\rho_{n \prime}=\frac{\rho_{m^{\prime}}-\min \rho_{m^{\prime}}}{\max \rho_{m^{\prime}}-\min \rho_{m \prime}}$

The most appropriate threshold value of $\rho_{n}$, is then determined by the OTSU (Otsu, N., 1979) method and the $\rho_{\text {mask }}$ mask is created so that the values above the threshold value of the $\rho_{n}$, matrix are marked as 1 and the values below it are 0 . Finally, all clutter are removed by multiplying the original image with the mask as given below.

$F i l_{S A R}=\rho_{\text {mask }} \cdot|\rho|$

\section{RESULTS: MMW GB-SAR MEASUREMENTS}

In order to conduct MMW GB-SAR imaging experiments, the measurement system given in Fig. 2a was constructed in the International Laboratory for High 
Technology (ILHT) in the TUBITAK Marmara Research Center. The system consists of two MMW horn antennas (WR-10), a Vector network analyzer (VNA E8362B) and computer to control the mobile platform. Measurements were carried out in the frequency range of 75 - $110 \mathrm{GHz}$. Half-power beam width of the antennas was measured as $24^{0}$ at $100 \mathrm{GHz}$. The dimensions of the materials used as target are given in Fig. 2b. Since the main purpose of this study was to eliminate unwanted clutter in GB-SAR images, the targets in both experiments were placed on the back side of the Electromagnetic (EM) absorber material. Since the back surface of the EM absorber material has a very high reflectivity, an artificial clutter was created and the performance of the proposed filter could be better observed.

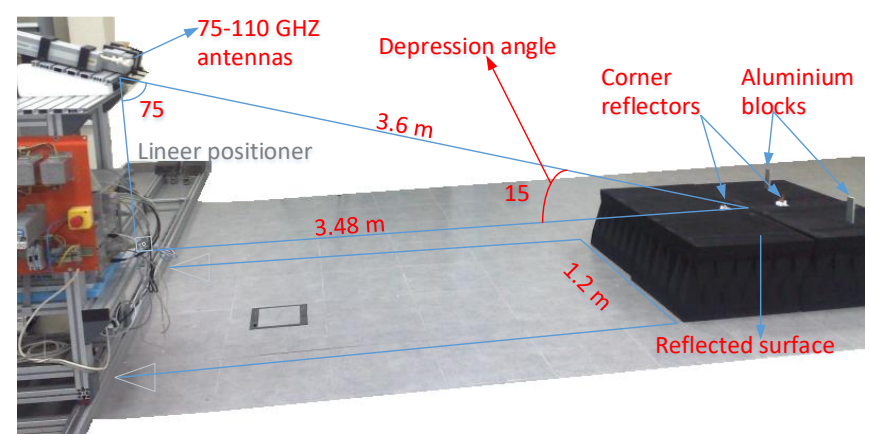

(a)

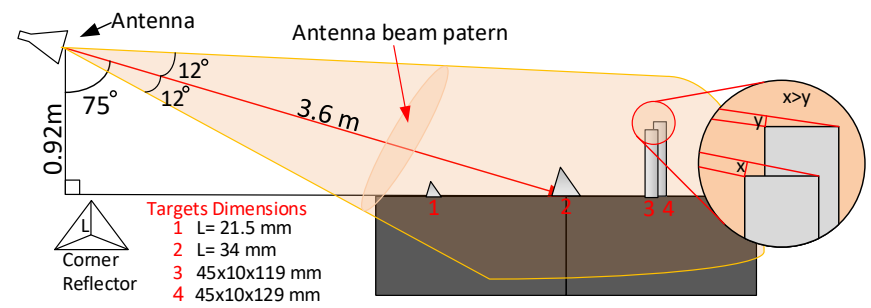

(b)

Figure 2. MMW GB-SAR imaging experimental setup; a) Linear positioner, antennas and an image of the targets, b) dimensions of the targets and geometry of the experimental setup

In the experiments, the VNA mounted on the mobile platform gathered backscatter data at a total of 301 points along a $1.2 \mathrm{~m}$ synthetic aperture. At each data collection point, the frequency of the VNA was altered between $75 \mathrm{GHz}$ and $81 \mathrm{GHz}$ in 201 steps. Thus, each GBSAR data had been expressed as a 301x201 matrix. The distance of the reflective surface where the targets are deployed to the platform was determined as $3 \mathrm{~m}$, and the altitude of the antennas was set as $0.92 \mathrm{~m}$ as shown in Fig. 2b.

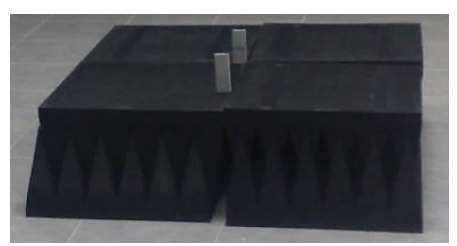

(a)

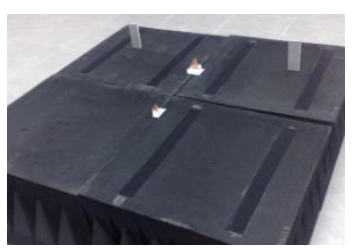

(b)
Figure 3. Targets used in measurements; a) $1^{\text {st }}$ Measurement, b) $2^{\text {nd }}$ Measurement

\subsection{Experiment 1}

In the first measurement, 2 aluminum blocks shown in Fig. 3a were used as targets. The first block was located $3 \mathrm{~m}$ from the antennas, while the second block was located $3.8 \mathrm{~m}$ from the antennas. Then, scanning was performed with the given measurement parameters. As it can be seen in Fig. 4, it is impossible to make any inferences about targets from the raw GB-SAR image.

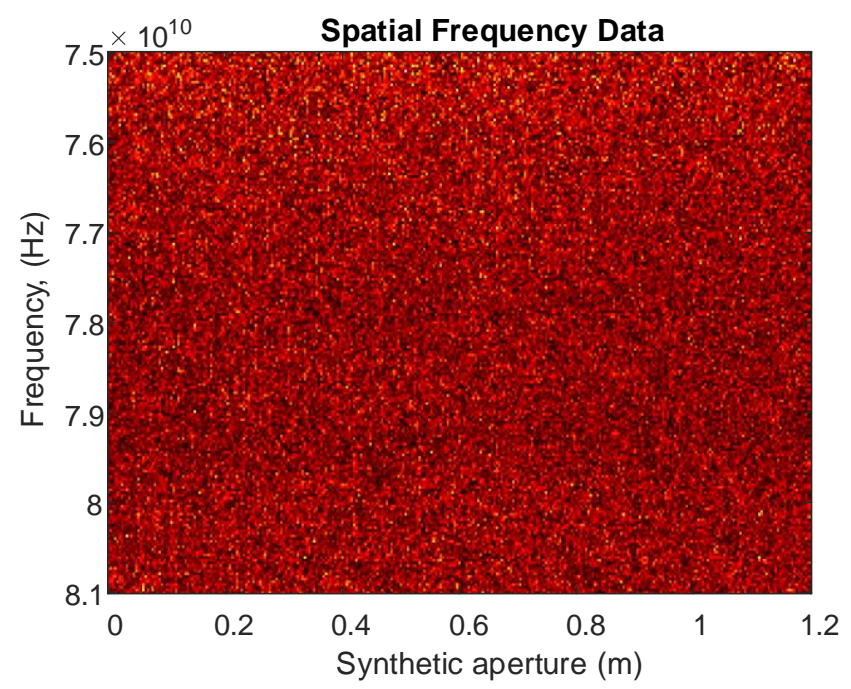

Figure 4. Raw GB-SAR data obtained from 2 aluminum blocks.

Since a highly reflective material was used as the background, the gathered row SAR image contains very high clutter. To apply the recommended filtering method, the image in Fig. 4 is primarily reconstructed with BPA and the amplitude of the complex SAR image is shown in Fig. 5.

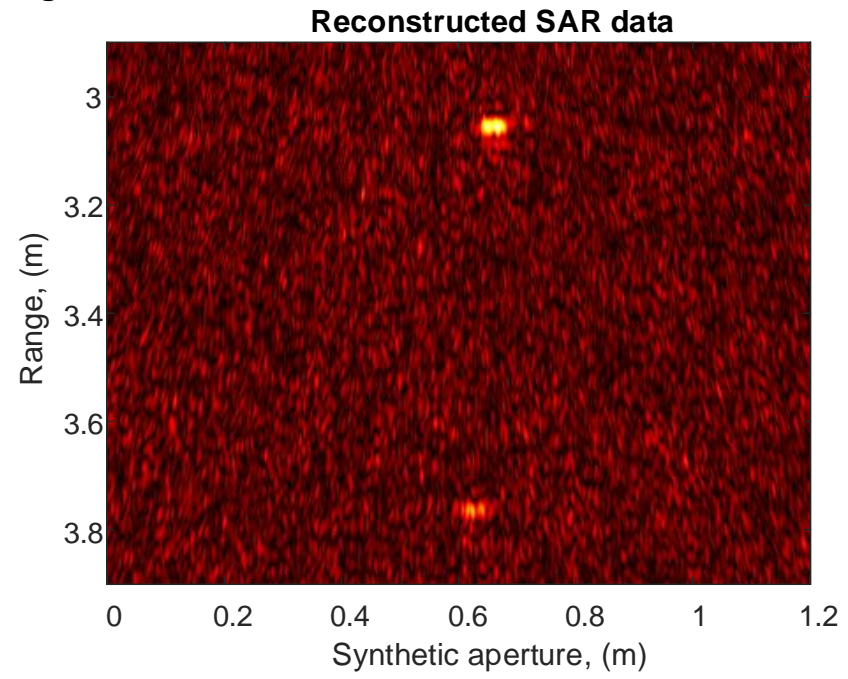

Figure 5. The SAR image of Experiment 1 reconstructed with $\mathrm{BPA}$

As seen in the Fig. 5, the metal block at $3 \mathrm{~m}$ can be distinguished after reconstruction, while the second block at $3.8 \mathrm{~m}$ has almost disappeared among the background clutter. In order to clean the clutter in the image, the recommended process steps in the second section are applied and $\rho_{n}$, is showed in Fig. 6. 


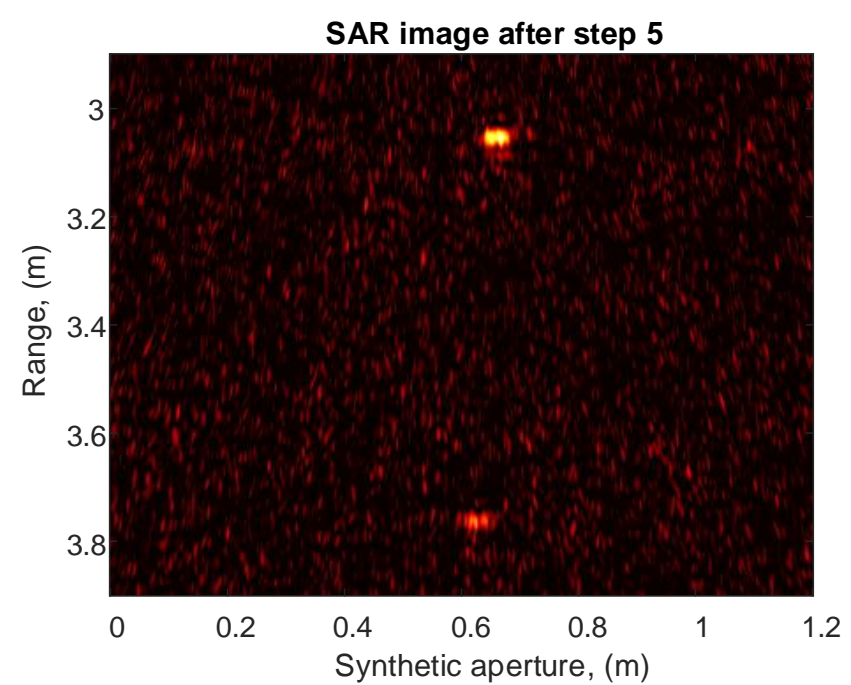

Figure 6. The image obtained after application of Eq. 5.

As can be seen in Fig. 6, the contrast in the SAR data has been increased and the target at $3.8 \mathrm{~m}$ has become a little more visible. To find the most appropriate threshold value, the OTSU method is applied to $\rho_{n \prime}$ and the threshold value is found as 0.0941 . A mask image (see Fig. 7) is then created where all items below the threshold value are 0 and items above the threshold are 1.

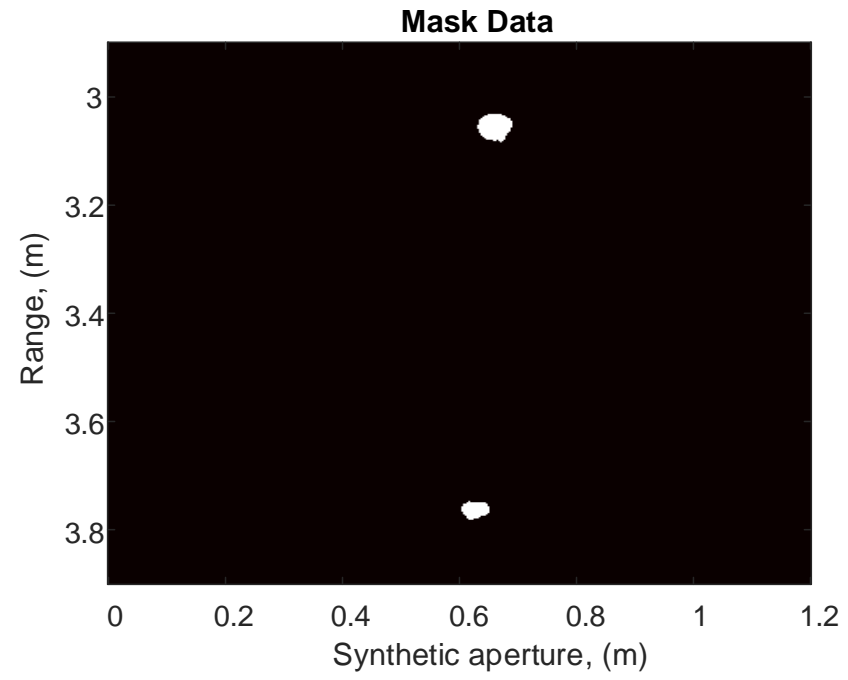

Figure 7. Mask image after application of the optimal thresholds found by OTSU's method.

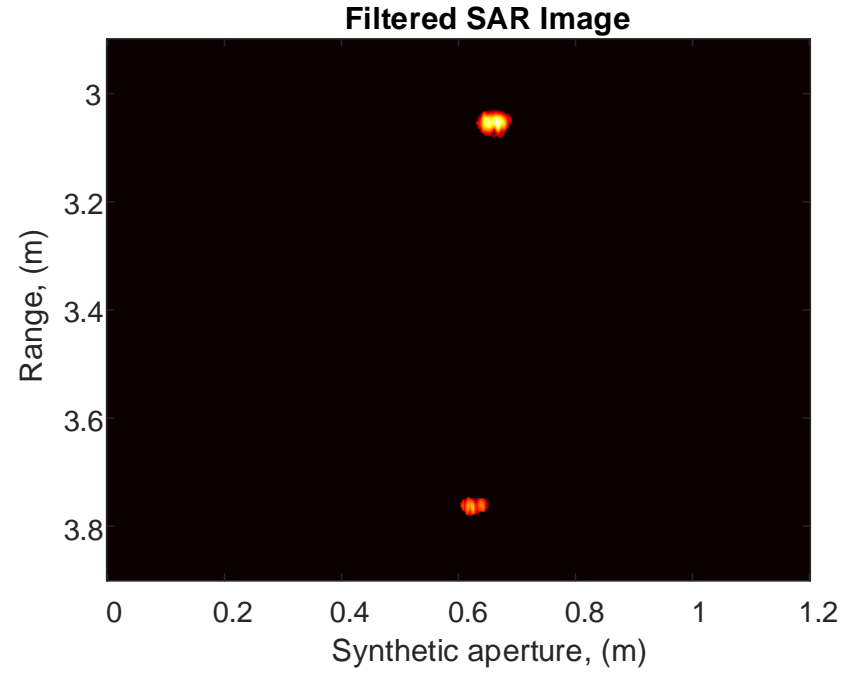

Figure 8. OTSU based filtered GB-SAR image
Then, the filtered image is obtained by multiplying the mask data given in Fig. 7 with the reconstructed raw GBSAR data. The result is seen in Fig. 8, and reveals that with the proposed technique, almost all clutter is completely removed and only the scattering signatures of the targets of interest are remained in the image. Thanks to the success of the proposed method, the target at $3.8 \mathrm{~m}$ is clearly displayed without any loss of information.

\section{2. $\quad$ Experiment 2}

In the second measurement, 4 targets are tried to be detected by adding 2 corner reflectors given in Fig. 3b. In this measurement, since the first corner reflector shown in Fig. 1 has very small dimensions, it is almost not seen in the reconstructed image in Figure 9.

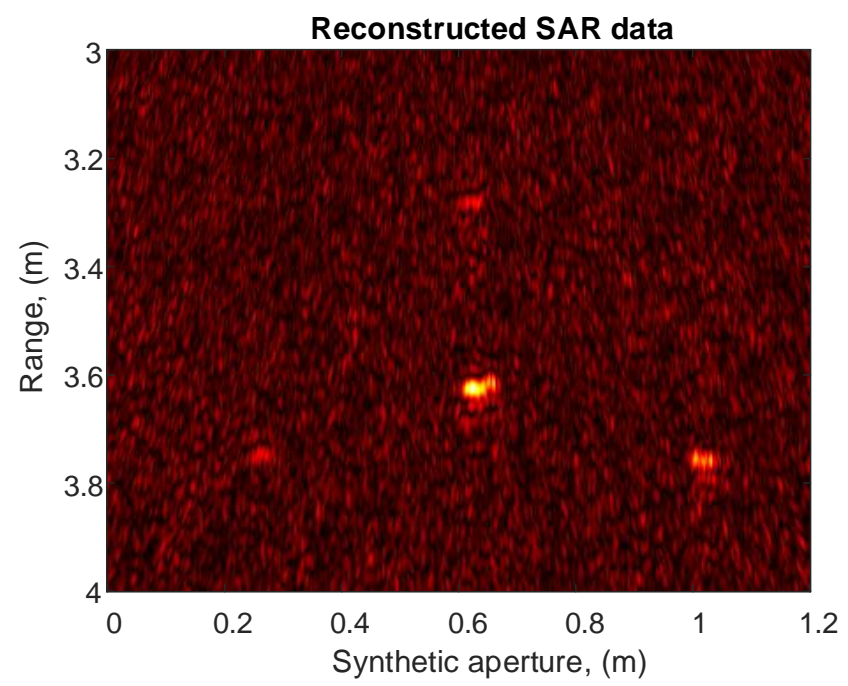

Figure 9. GB-SAR image, obtained from 2 aluminum blocks and 2 corner reflectors

The mask matrix obtained after applying the recommended filtering to the image in Figure 9 is shown in Fig. 10. In this measurement, the threshold value obtained with OTSU is found as 0.1020 .

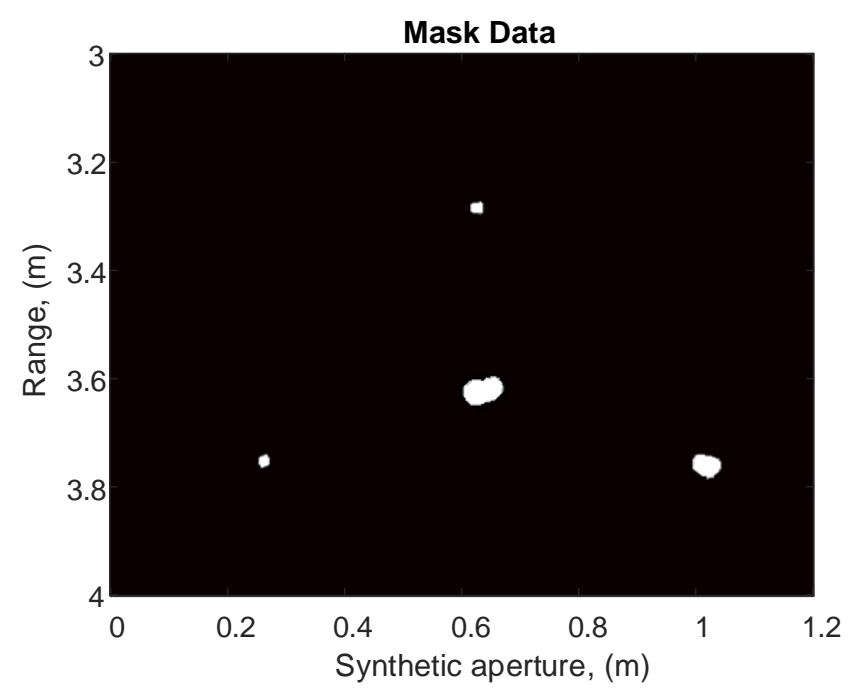

Figure 10. The created Mask image after thresholding found by OTSU method 
When the data reconstructed with BPA is multiplied with the mask matrix shown in Fig. 10, the filtered GBSAR image is given in Fig. 11.

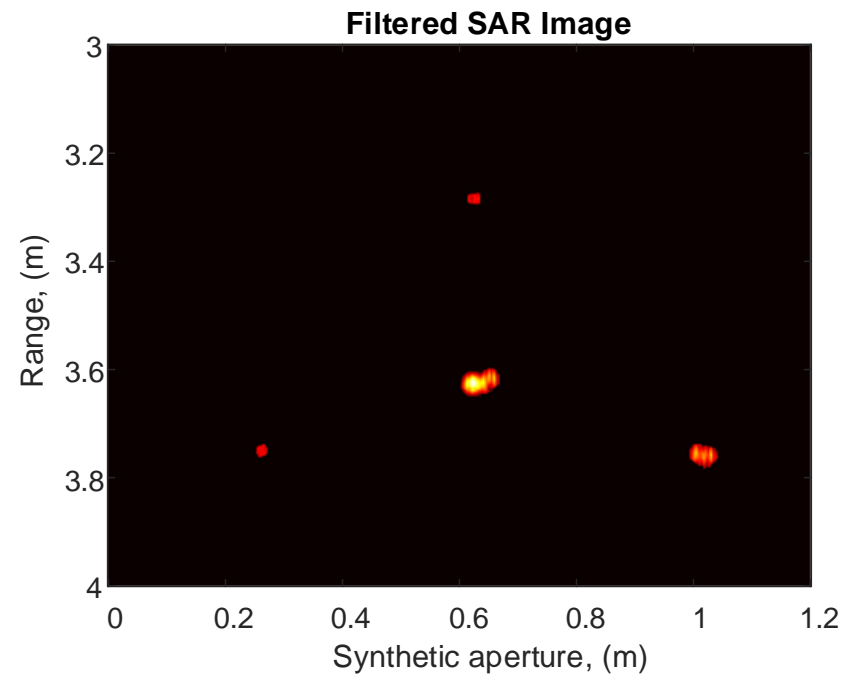

Figure 11. OTSU based filtered GB-SAR image of 4 targets

As seen in Fig. 11, owing to the suggested method, all the clutter is removed and the objects are obviously identified in their exact locations. Thanks to the filtering technique based on the OTSU method, since the targets are cleaned from clutter, there is no dynamic reduction in the filtered image. When Fig. 9 is examined, the small corner reflector and metal block are almost not visible. However, thanks to the applied technique, targets that could not be detected visually are clearly identified and no false target detection is realized. These results clearly demonstrate the performance of the presented technique in filtering highly dispersed data and are promising for further studies. When the results of both measurements are evaluated, it is seen that the threshold value of the first measurement is smaller than the second measurement. Since metal blocks with high reflectivity are closer to the SAR path in the first measurement, the signal to clutter value is higher than the second measurement. Therefore, with a lower threshold value compared to the second measurement, the clutter was completely cleaned. These results clearly demonstrate that the OTSU method can be effectively applied to raw SAR data other than RGB images.

\section{CONCLUSION}

In this study, an effective clutter removal method that can be applied in all kinds of SAR images is proposed. The most suitable threshold values are determined by using the OTSU method, which can be applied directly to raw data without converting the GB-SAR data into any RGB image. According to the found threshold value, clear GBSAR images are obtained by masking processes. Thanks to the proposed technique, all targets are successfully detected without any loss of data regarding the target images. These results obtained in MMW measurements with very high clutter show that the proposed technique can be applied effectively to further SAR data. Since the clutter is mostly eliminated with the proposed technique, the performance of target detection algorithms such as machine learning-based SAR, ISAR or GPR [Ozkaya, 2020; Ozkaya and Seyfi, 2018] can be increased.

\section{ACKNOWLEDMENT}

The authors would like to thank Mr. Atilla Unal and Mr. Mustafa Tekbas for their assistance during the experiments. The authors also thank the ILHT for providing measurement facilities.

\section{Author contributions}

Enes Yigit: Developing the technique, Execution of experiments, Writing-Original draft preparation; Şevket Demirci: Execution of experiments, Writing-reviewing, and editing; Caner Özdemir: Conceptualization, Evaluation and presentation of results, reviewing and editing of the manuscript.

\section{Conflicts of interest}

The authors declare no conflicts of interest.

\section{REFERENCES}

Beasley P, Binns G, Hodges R \& Badley R (2004). A Millimetre Wave Radar for Airport Runway Debris Detection. First Europen Radar Conference, Amsterdam, Nerherlands, 261-264.

Conte E, Longo M, Lops M \& Ullo S L (1991). Radar detection of signals with unknown parameters in Kdistributed clutter. IEE Proc F Radar Signal Process $138,131-138$.

Demirci S, Cetinkaya H, Yigit E, Ozdemir C \& Vertiy A A (2012). A Study on Millimeter-Wave Imaging of Concealed Objects: Application Using BackProjection Algorithm. Progress In Electromagnetics Research, 128, 457-477.

Demirci S, Ozdemir C, Akdagli A \& Yigit E (2008). Clutter Reduction In Synthetic Aperture Radar Images With Statistical Modeling: An Application To Mstar Data. Microwave And Optical Technology Letters.50 (6).

Engin E, Çiftçioğlu B, Özcan M \& Tekin İ (2007). High Resolution Ultrawideband Wall Penetrating Radar. Microwave and optical technology letters, 49(2), 320-325.

Freitas C C, Frery A C \& Correia A (2005). The polarimetric $\mathrm{G}$ distribution for SAR data analysis. Environmetrics 16, 13-31.

Gomez-Dans J L, Quegan S \& Bennett J C (2006). Indoor C-Band Polarimetric Interferometry Observations of a Mature Wheat Canopy. IEEE transactions on geoscience and remote sensing, 44(4), 768-777.

Işıker H \& Özdemir C (2019). A Multi-Thresholding Method Based on Otsu's Algorithm for the Detection of Concealed Threats in Passive Millimeter-Wave Images. Frequenz, 73, issue 5-6, 179-187.

Ișıker H, Özdemir C, Unal İ (2015). Millimeter-Wave Band Radiometric Imaging Experiments for the Detection of Concealed Objects. IEEE Radar Conference, Johannesburg, South Africa, 27-30 October.

Işıker H, Ünal İ, Tekbaș M, Özdemir C (2018). An AutoClassification Procedure for Concealed Weapon Detection in Millimeter-Wave Radiometric Imaging Systems. Microwave Opt. Tech. Letters, 60(3), 583594.

Jaeger I, Zhang L, Stiens J, Sahli H \& Vounckx R (2007). Millimeter Wave Inspection of Concealed Objects. Microwave and optical technology letters, 49(11), 2733-2737. 
Khoukhi H, Filali Y, Yahyaouy A, Sabri M A, Aarab A (2019). A hardware Implementation of OTSU Thresholding Method for Skin Cancer Image Segmentation. 2019 International Conference on Wireless Technologies, Embedded and Intelligent Systems (WITS),

Lu J \& Hu R (2012). A new image segmentation method based on Otsu method and ant colony algorithm," 2012 International Conference on Computer Science and Information Processing (CSIP), Xi'an, China, 2012 , pp. 767-769 doi: 10.1109/CSIP.2012.6308966.

Otsu N (1979). A Threshold Selection Method from GrayLevel Histograms. IEEE transactions on systems, man, and cybernetics, 9(1), 62-66

Ozkaya U (2020). Automatic Target Recognition (ATR) from SAR Imaginary by Using Machine Learning Techniques, European Journal of Science and Technology, 165-169

Ozkaya U, Seyfi L (2018). Deep dictionary learning application in GPR B-scan images. Signal, Image and Video Processing, 12, 1567-1575

Pieraccini M, Luzi G, Mecatti D, Noferini L \& Atzeni C (2006). Ground-Based Sar for Short and Long Term Monitoring of Unstable Slopes. 3rd Europen Radar Conference, Manchester, United Kindgom, 92-95.
Sabanci K, Yigit E, Toktas A, Kayabasi A (2018). A Huedomain filtering technique for enhancing spatial sampled compressed sensing-based SAR images. IET Radar, Sonar\&Navigation, 13(3), 357-367.

Toktas A, Yigit E, Sabanci K, Kayabasi A. (2017). CFAR based morphological filter design to remove clutter from GB-SAR images: An application to real data. Microw Opt Technol Lett. 59, 2685 -2692.

Yigit E, Demirci S, Ozdemir C, Tekbas M (2013). Shortrange ground-based synthetic aperture radar imaging: performance comparison between frequency-wavenumber migration and backprojection algorithms. J. Appl. Rem. Sens. 7(1).

Yigit E, Demirci S, Unal A, Ozdemir C \& Vertiy A (2012). Millimeter-Wave Ground-Based Synthetic Aperture Radar Imaging for Foreign Object Debris Detection: Experimental Studies at Short Ranges. Journal of Infrared, Millimeter, and Terahertz Waves, 33(12), 1227-1238.

Yigit E, Ozkaya U, Ozturk S (2020). Enhancement of Near Field GB-SAR Image Quality Using Beamwidth Filter. European Journal of Science and Technology Special Issue, 480-487.

(C) Author(s) 2022. This work is distributed under https://creativecommons.org/licenses/by-sa/4.0/ 